\title{
A dual strategy to improve psychotic patients' compliance using sustained release quetiapine oral disintegrating tablets
}

\author{
AHMED REFAAT* \\ MAGDA SOKAR \\ FATMA ISMAIL \\ NABILA BORAEI \\ Pharmaceutics Department \\ Faculty of Pharmacy \\ Alexandria University, Alexandria \\ Egypt
}

Accepted February 21, 2016 Published online June 7, 2016

\begin{abstract}
Quetiapine (QT) is a short acting atypical antipsychotic drug effective in schizophrenia and bipolar disorder. This study aims at designing a novel dosage form of sustained release taste-masked QT orally disintegrating tablets (ODTs) based on solid lipid micro-pellets (SLMPs). QT SLMPs were prepared using the hot melt extrusion technique and utilizing three lipid carriers: Compritol, Precirol and white beeswax either alone or in mixtures. They showed sustained QT release and a taste masking effect. The selected QT SLMP was further blended with an aqueous solution containing polyvinylpyrollidone (2.5\%), croscarmellose sodium (2\%) and mannitol (50\%); it was then lyophilized into ODT in a mass ratio of 1:2, respectively. ODTs containing QT SLMPs showed: average wetting time (40.92 s), average oral disintegration time (21.49 s), average hardness $(16.85 \mathrm{~N})$ and also imparted suitable viscosity to suspend pellets during the lyophilization process. In conclusion, lyophilization is a promising technique for the formulation of multiparticulate systems into ODTs.
\end{abstract}

Keywords: solid lipid micro pellets, multiparticulate, quetiapine, orally disintegrating tablets

Quetiapine is a short-acting atypical neuroleptic drug that was first approved by FDA in 1997 under the name of Serquel ${ }^{\circledR}$ for the treatment of schizophrenia and bipolar disorder (1). Quetiapine showed pharmacological characteristics distinct from classic neuroleptics that function primarily via blockade of dopamine $\mathrm{D}_{2}$ receptors where quetiapine very rapidly disassociates from the $\mathrm{D}_{2}$ receptor, minimizing the risk of side effects such as pseudoparkinsonism and elevations in prolactin (2). Quetiapine also acts as a partial agonist at $5-\mathrm{HT}_{1 \mathrm{~A}}$ receptors, so it is used in combination with antidepressants for the treatment of major depressive disorders (3).

Adherence to treatment in psychiatric as well as in any other chronic conditions is often suboptimal. A high proportion of relapses are due to non-adherence to prescribed treatment. Orally disintegrating tablets (ODTs) were developed with the aim to improve

\footnotetext{
*Correspondence; e-mail: A.R.Ahmed@alexu.edu.eg; Ahmedref3at.ph@gmail.com
} 
patient compliance due to their fast oral disintegration and ease of swallowing (4). Risperidal ${ }^{\circledR} \mathrm{M}$-Tab (risperidone ODTs) increased greatly patient acceptance of daily therapy. Since most antipsychotics are unpalatable, orally disintegrating drug delivery systems usually contain the active pharmaceutical ingredient in a taste-masked form (5).

Another approach, to enhance schizophrenic compliance with antipsychotic treatment, is through formulation of the drug in sustained release dosage forms (3). Quetiapine shows $100 \%$ bioavailability following oral dosing with a 6-hour biological half-life. Moreover, it has moderate solubility in water (6), so its pharmacokinetic and physicochemical properties make it a good candidate to be formulated into sustained release delivery systems. Sustained release delivery systems can be single-unit or multi-particulate systems. Multiple-unit systems include various subunits, namely granules, beads, pellets, micropellets, mini-tablets, microspheres, microcapsules, liposomes and nanoparticles (7).

Multiple-unit systems share several advantages over single-unit systems. Their small size enables them to be well distributed along the gastrointestinal tract resulting in a reduction of local drug concentration and avoidance of dose dumping, which may occur due to failure of sustained release single-unit dosage forms $(8,9)$. Inter- and intra-individual variations in bioavailability caused by food effects are reduced. Moreover, coated multiple units have more rapid transit time compared to enteric-coated tablets, avoiding premature drug release from the enteric dosage form, reducing drug degradation or irritation of gastric mucosa (10). Other advantages of multiple-unit systems include the ease of administration of incompatible drugs by separating them in different multi-particulates and modulation of release kinetics.

An ODT containing a sustained release multi-particulate system of QT would be a promising delivery system for psychotic patients because compliance would be enhanced through reduced dosing frequency, increased ease of administration and taste masking of quetiapine.

This study is aimed at preparation and characterization of quetiapine lipid micropellets as a taste masked sustained release delivery system and their incorporation into lyophilized oral disintegrating matrices that would enhance psychotic patient compliance.

\section{EXPERIMENTAL}

Quetiapine fumarate (QT) was purchased from Radiant Co., India. Compritol ATO 888 and Precirol ATO 5 were kindly provided by Gattefossé, France. White beeswax 8108 (Cera Alba), polyvinylpyrollidone (PVP K25), croscarmellose sodium (CCNa) and mannitol were obtained as gifts from Pharco Pharmaceuticals Co., Egypt. Acetonitrile, methanol, hydrochloric acid and ethanol (Fisher HPLC grade) were purchased from Cornall Labs, Egypt. Sodium triphosphate and potassium dihydrogen phosphate were purchased from Winilab laboratory chemicals, UK. Orthophosphoric acid was purchased from El-Nasr Pharmaceutical Chemicals Co., Egypt.

\section{High performance liquid chromatography assay of QT}

High performance liquid chromatography (HPLC) assay, proposed by the supplier company, was performed with slight modification. The HPLC system (Agilent 1100 supplied with an LC pump, Online Series vacuum degasser, autosampler, UV detector) was 
equipped with a reversed phase C18 column Hypersil BDS $(100 \times 4.6 \mathrm{~mm}$ i.d., $5 \mu$ particle size). The mobile phase used was $0.05 \mathrm{~mol} \mathrm{~L}^{-1}$ potassium dihydrogenphosphate buffer and acetonitrile in a ratio 1:4, at a flow rate of $1 \mathrm{~mL} \mathrm{~min}{ }^{-1}$. The injection volume was $20 \mu \mathrm{L}$ and the peaks were detected spectrophotometrically at $\lambda_{\max } 225 \mathrm{~nm}$. All runs were performed at controlled room temperature $\left(20^{\circ} \mathrm{C}\right)$.

Quetiapine calibration curves in both $0.1 \mathrm{~mol} \mathrm{~L}^{-1} \mathrm{HCl}$ and buffer $\mathrm{pH} 6.8$ were prepared over a concentration range of $40-120 \mu \mathrm{g} \mathrm{mL}^{-1}$.

Validation parameters of the HPLC assay were limited to linearity, inter-day and intra-day precision data.

Preparation of quetiapine solid lipid micro-pellets (QT SLMPs)

A modified hot melt extrusion (HME) technique was adopted for the preparation of QT SLMPs (12). A total mass (4 g) consisting of a mixture of quetiapine and lipid carriers was allowed to melt under continuous stirring at $90{ }^{\circ} \mathrm{C}$ on a thermostatically controlled water bath (Kotterman, Germany). Lipid carriers, namely Compritol ATO 888, Precirol ATO 5 or white beeswax were used either alone or in combination, as shown in Table I.

The molten mass was then allowed to congeal $\left(\sim 30^{\circ} \mathrm{C}\right)$, under continuous stirring, to a mass of plasticity suitable enough to be forced through a sieve of $1.25-\mathrm{mm}$ mesh size (Retsch, Germany). The extruded rods were then forced again through a sieve of $0.75-\mathrm{mm}$ mesh size using a flexible spatula to obtain micro-pellets. The size fraction of $0.5-1 \mathrm{~mm}$ was used in all testing.

\section{Characterization of QT SLMPS}

Scanning electron microscope (SEM). - The morphology and surface characteristics of QT SLMPs were examined using a scanning electron microscope (model JEM-100S, JEOL, Japan). Samples of QT loaded micropellets were mounted onto metal stubs using double sided adhesive tape. The stubs were then coated with gold using a sputter coater before being viewed under SEM.

Table I. Composition of QT solid lipid micropellets

\begin{tabular}{ccc}
\hline Pellet code & Lipid & Ratio of QT to lipid $(\mathrm{m} / \mathrm{m})$ \\
\hline C1 & & $1: 1$ \\
C2 & Compritol & $1: 2$ \\
C3 & & $1: 3$ \\
P1 & Precirol & $1: 1$ \\
P2 & & $1: 2$ \\
P3 & & $1: 3$ \\
B1 & & $1: 1$ \\
B2 & White beeswax & $1: 2$ \\
B3 & & $1: 3$ \\
PB & Precirol and beeswax & $1: 1: 1$ \\
\hline
\end{tabular}


Differential scanning calorimetry (DSC). - A differential scanning calorimeter (Perkin Elmer instruments, USA) was used to determine the crystalline state of each individual component, physical mixture (PM) and the QT SLMPs.

The instrument was calibrated with indium, dry nitrogen was used as a carrier gas with a flow rate of $25 \mathrm{~mL} \mathrm{~min}{ }^{-1}$ at a scan speed of $10^{\circ} \mathrm{C} \mathrm{min}^{-1}$ up to $350{ }^{\circ} \mathrm{C}$. Sample mass was $4 \mathrm{mg}$ for free QT and $8 \mathrm{mg}$ for QT loaded micropellets and PM samples containing an equivalent amount of the drug.

Fourier transform infrared spectroscopy (FTIR). - Samples of QT, Compritol, Precirol, white beeswax and QT loaded micropellets along with their corresponding PM were mixed with $\mathrm{KBr}$ and compressed into discs. The scanning range was 4000 to $400 \mathrm{~cm}^{-1}$ to investigate the drug-lipid interaction. FTIR spectra were obtained using a Perkin Elmer spectrum RXIFT-IR system, USA.

Drug content. - Drug content of QT SLMPs was evaluated using the hot acid extraction method. An accurate amount of SLMPs equivalent to $10 \mathrm{mg}$ of QT, was weighed and added to a $100-\mathrm{mL}$ volumetric flask. Twenty milliliters of $0.1 \mathrm{~mol} \mathrm{~L}^{-1} \mathrm{HCl}$ were then added to the QT SLMPs. The suspension obtained was sonicated (Ultrasonic Cleaner Eumex, Kwun Wah International Ltd, Hong Kong, China) at $65^{\circ} \mathrm{C}$ for 30 minutes, then the volume was completed to $90 \mathrm{~mL}$ with $0.1 \mathrm{~mol} \mathrm{~L}^{-1} \mathrm{HCl}$ and left in a water bath at $90{ }^{\circ} \mathrm{C}$ for 10 minutes to ensure complete melting of the lipophilic phase releasing any unextracted QT in the form of QT HCl.

Suspension was then left to cool to room temperature and the volumes were adjusted to $100 \mathrm{~mL}$ in another volumetric flask, followed by 10 minutes of sonication at room temperature $\left(\sim 25^{\circ} \mathrm{C}\right)$. An appropriate volume was filtered using a $0.45-\mu$ membrane filter to remove the congealed lipophilic phase. Filtrates were suitably diluted with $0.1 \mathrm{~mol} \mathrm{~L}^{-1} \mathrm{HCl}$ and analyzed using the previously mentioned HPLC assay method.

\section{In vitro release studies}

Studies were carried out on QT SLMPs equivalent to $150 \mathrm{mg}$ QT using a USP dissolution apparatus II (Copley Scientific, UK). The dissolution medium consisted of $730 \mathrm{~mL} 0.1$ mol L-1 $\mathrm{HCl}$ for 2 hours, followed by $\mathrm{pH} 6.8$ buffer, adjusted by the addition of $270 \mathrm{~mL}$ of $0.2 \mathrm{~mol} \mathrm{~L}^{-1}$ trisodium phosphate for 12 hours (13). The stirring rate was $50 \mathrm{rpm}$ and the release media temperature was maintained at $37 \pm 0.5^{\circ} \mathrm{C}$. Samples $(5 \mathrm{~mL})$ were withdrawn at predetermined time intervals $(1,2,4,6,8,10,12$ hours), compensated with fresh dissolution medium and assayed using the HPLC method.

\section{Release kinetics evaluation}

Kinetics of quetiapine released from the prepared micropellets was examined based on the magnitude of the coefficient of determination $\left(R^{2}\right)$ obtained after application of zero order, first order and Higuchi models using the Add-In DDS solver software.

\section{Release stability study}

The release behavior of the selected QT SLMPs was assessed over a one-year study period. QT SLMPs in airtight plastic containers were stored in a desiccator of silica beads 
A. Refaat et al.: A dual strategy to improve psychotic patients' compliance using sustained release quetiapine oral disintegrating tablets, Acta Pharm. 66 (2016) 515-531.

Table II. Composition of different ODTs' lyophilized matrices

\begin{tabular}{ccccc}
\hline $\begin{array}{c}\text { Matrix } \\
\text { code }\end{array}$ & $\begin{array}{c}\text { PVP K25 } \\
(\%)\end{array}$ & $\begin{array}{c}\text { Mannitol } \\
(\%)\end{array}$ & $\begin{array}{c}\text { Croscarmellose } \\
\text { sodium (\%) }\end{array}$ & $\begin{array}{c}\text { Water } \\
(\%)\end{array}$ \\
\hline $\mathrm{M}_{1}$ & 2.5 & - & - & 50.5 \\
$\mathrm{M}_{2}$ & 5.0 & 45.0 & - & 48.0 \\
$\mathrm{M}_{3}$ & 10.0 & - & - & 43.0 \\
$\mathrm{M}_{4}$ & 2.5 & - & - & 45.5 \\
$\mathrm{M}_{5}$ & 5.0 & 50.0 & 2 & 43.0 \\
$\mathrm{M}_{6}$ & 10.0 & - & - & 38.0 \\
$\mathrm{M}_{7}$ & 2.5 & - & - & 35.5 \\
$\mathrm{M}_{8}$ & 5.0 & 60.0 & - & 33.0 \\
$\mathrm{M}_{9}$ & 10.0 & - & - & 28.0 \\
\hline
\end{tabular}

at room temperature $\left(\approx 25^{\circ} \mathrm{C}\right)$ and $25 \%$ relative humidity. QT release profiles were obtained after 6 and 12 months of storage and then compared to the freshly prepared SLMPs.

\section{Preparation of lyophilized matrices for ODT}

The composition of different ODT matrices is given in Table II. PVP K-25 and croscarmellose were dissolved in specified amounts of water in a small glass mortar, and then mannitol was added and blended to form a homogenous thick dispersion. The amount of $1 \mathrm{~mL}$ dispersion was dosed into each pocket of a polyvinylchloride (PVC) blister pack of $15 \mathrm{~mm}$ diameter and $5 \mathrm{~mm}$ depth. The tablet blister packs were frozen at $-20^{\circ} \mathrm{C}$ and then lyophilized (Novalyphe-NL 500 freeze dryer) with a condenser temperature of $-45^{\circ} \mathrm{C}$ and a pressure of $7 \times 10^{-2}$ mbar.

\section{Evaluation of prepared tablet matrices}

Viscosity measurement of liquid blends of matrices. - Viscosities of $100 \mathrm{~mL}$ of matrix liquid blends were measured using a Brookfield digital viscometer (Harlow, UK) at room temperature. The rotational speed of the spindle was set at $100 \mathrm{rpm}$. Each reading was done in triplicate.

Physical characteristics of prepared lyophilized matrices. - Matrix thickness and mass were determined using a micrometer (Moore and Wright Ltd, UK) and a digital balance, respectively. The breaking force (hardness) was measured using a hardness tester (Erweka Hardness Tester, GmbH, Germany).

\section{Determination of disintegration time}

Oral disintegration time. - Six healthy male volunteers participated in the trial to assess the oral disintegration time of lyophilized matrices, after giving their written consent. Volunteers were asked to rinse their mouth with distilled water and matrix tablets were 
placed on their tongues. They were allowed to move the tablet against their upper palate, which caused a gentle tumbling action on the tablet without biting or tumbling it from side to side. The time needed for complete disintegration was then recorded (14).

Wetting test (modified USP disintegration test). - A piece of tissue paper, folded twice was placed in a small Petri dish containing $6 \mathrm{~mL}$ of water. The matrix tablet was then placed on the wetted paper and the time for complete wetting was recorded (15).

Scanning electron microscopy. - The surface and inner structural morphology of the freeze-dried matrices were examined by scanning electron microscopy. Thin horizontal and vertical samples of tablet matrices were prepared by cutting them with a surgical blade. The samples were placed onto double-sided adhesive strips on aluminum stubs and coated with a thin layer of gold using a sputter coater at $20 \mathrm{~mA}$ for three minutes and then examined by SEM.

\section{Preparation of quetiapine oral disintegrating tablets}

Two different QT ODT formulations were prepared by blending the selected QT SLMP with the selected tablet matrix (1:2, respectively) followed by lyophilization (Table III). Dispersion was dosed in each pocket of a PVC pack to fill a blister of $13 \mathrm{~mm}$ diameter, 3 $\mathrm{mm}$ depth in case of $\mathrm{F}_{1}$ and $15 \mathrm{~mm}$ diameter, $5 \mathrm{~mm}$ depth for $\mathrm{F}_{2}$ to get two tablet formulations equivalent to $50 \mathrm{mg}$ and $100 \mathrm{mg}$ quetiapine, respectively.

The tablet blister packs were directly frozen at $-20^{\circ} \mathrm{C}$ for $24 \mathrm{~h}$ and then lyophilized applying condenser temperature of $-45^{\circ} \mathrm{C}$ with $7 \times 10^{-2}$ mbar pressure.

\section{Evaluation of QT ODTs}

Evaluation of prepared ODTs. - Tablet thickness and hardness were measured. Mass uniformity test was also performed according to the USP method and oral disintegration and wetting times were measured.

In vitro release studies. - USP dissolution apparatus II was used. The dissolution medium, stirred at $50 \mathrm{rpm}$, consisted of $730 \mathrm{~mL} 0.1 \mathrm{~mol} \mathrm{~L}^{-1} \mathrm{HCl}$ for 2 hours, followed by a buffer of pH 6.8 adjusted by the addition of $270 \mathrm{~mL}$ of $0.2 \mathrm{~mol} \mathrm{~L}^{-1}$ trisodium phosphate, for 12 hours (13). Temperature of the release media $\left(0.1 \mathrm{~mol} \mathrm{~L}^{-1} \mathrm{HCl}\right.$ and $0.2 \mathrm{~mol} \mathrm{~L}^{-1}$ trisodium phosphate) was maintained at $37 \pm 0.5^{\circ} \mathrm{C}$. Samples $(5 \mathrm{~mL})$ were withdrawn at predetermined time intervals, compensated with fresh dissolution medium and assayed using the above mentioned HPLC method.

Table III. Composition of quetiapine oral disintegrating tablet formulations

\begin{tabular}{lcc}
\hline Ingredients & \multicolumn{3}{c}{ Formulation code } \\
\hline & $\mathrm{F}_{1}$ & $\mathrm{~F}_{2}$ \\
\hline QT micropellets & $150 \mathrm{mg}$ (equivalent to $50 \mathrm{mg} \mathrm{QT})$ & $300 \mathrm{mg}$ (equivalent to $100 \mathrm{mg}$ QT) \\
Matrix & $300 \mathrm{mg}$ & $600 \mathrm{mg}$ \\
\hline
\end{tabular}


A. Refaat et al.: A dual strategy to improve psychotic patients' compliance using sustained release quetiapine oral disintegrating tablets, Acta Pharm. 66 (2016) 515-531.

\section{RESULTS AND DISCUSSION}

\section{Preparation of QT SLMP}

Lipids and/or waxes as drug carriers in oral delivery systems have attracted researchers' interest since they are biodegradable and physiologically non-toxic $(12,16)$. Moreover, lipids are reported to achieve sustained release and a taste masking effect on drugs at a relatively low cost (16). In the present study, Compritol, Precirol and white beeswax were utilized to produce taste masked sustained release pellets. Emulsion congealing and hot extrusion method (HME) have been reported to produce SLMPs (12).

Quetiapine is a moderately water soluble drug with $\log P 2.65$, which makes it a poor candidate for incorporation into lipid carriers by the emulsion congealing method since it would be retained in water and the produced lipid pellets would show poor QT entrapment efficiency. On the other hand, the HME method can be considered as a good candidate method to incorporate QT into lipid carriers. HME method was suggested to prepare sustained release QT SLMPs due to its simplicity, for molten polymers can function as drug depots and drug release retardants upon cooling and solidification. Moreover, solvents and water are not necessary, which reduces the number of processing steps and eliminates time consuming drying steps (12).

The yield of different QT SLMPs ranged from 74 to $92 \%$. The observed loss is attributed to the loss of lipophilic matrices to the meshes of the sieves and vessel walls during formulation caused by melting succeeded by congealing to those surfaces. Such loss amounted to the highest value of $26 \%$. However, in large scale production, the different steps of the HME technique (mixing, melting, homogenizing and shaping) are carried out on a single machine. This offers more opportunities for automation of the production process, allows a high throughput, limits material loss and yields matrices with excellent homogeneity.

Higher yield values were observed for compritol and beeswax and, to a lesser extent, for precirol. This can be attributed to the rapid congealing of precirol (melting point $61^{\circ} \mathrm{C}$ ) with promoting loss of some of the lipid sticking to the walls of the vessel and mesh during the congealing of the matrix to micro-pellets.

The external morphology and surface characteristics of the prepared QT SLMPs (P1, $\mathrm{B} 1$ and $\mathrm{C} 1$, as representative ratios) were examined using the SE micrographs (Fig. 1). All the prepared pellets showed irregular ellipsoid shape, which is attributed to the manual process of extrusion. The surface of precirol micropellets (Fig. 1a) showed a highly irregular structure, with high dentations and grooves, while beeswax micropellets (Fig. 1b) showed smooth surfaces. The surface of compritol micropellets (Fig. 1c) exhibited surface indentations. These observations are of great impact on the release profile of each formula. Furthermore, crystals appeared on the surface of all the prepared micropellets that may be QT crystals.

DSC study was performed on QT loaded SLMPs $\left(\mathrm{C}_{1}, \mathrm{P}_{1}\right.$ and $\left.\mathrm{B}_{1}\right)$, their respective components alone and their physical mixtures. Free QT powder thermogram was characterized by a single sharp melting endothermic peak at $172{ }^{\circ} \mathrm{C}$. It revealed typical crystalline, anhydrous behavior. Thermograms of Compritol, beeswax and Precirol showed endothermic peaks at 70,65 and $61^{\circ} \mathrm{C}$, respectively, typical of their melting. It was observed that no shift in the endothermic peak of QT appeared in all thermograms of QT loaded SLMPs and 
A. Refaat et al.: A dual strategy to improve psychotic patients' compliance using sustained release quetiapine oral disintegrating tablets, Acta Pharm. 66 (2016) 515-531.

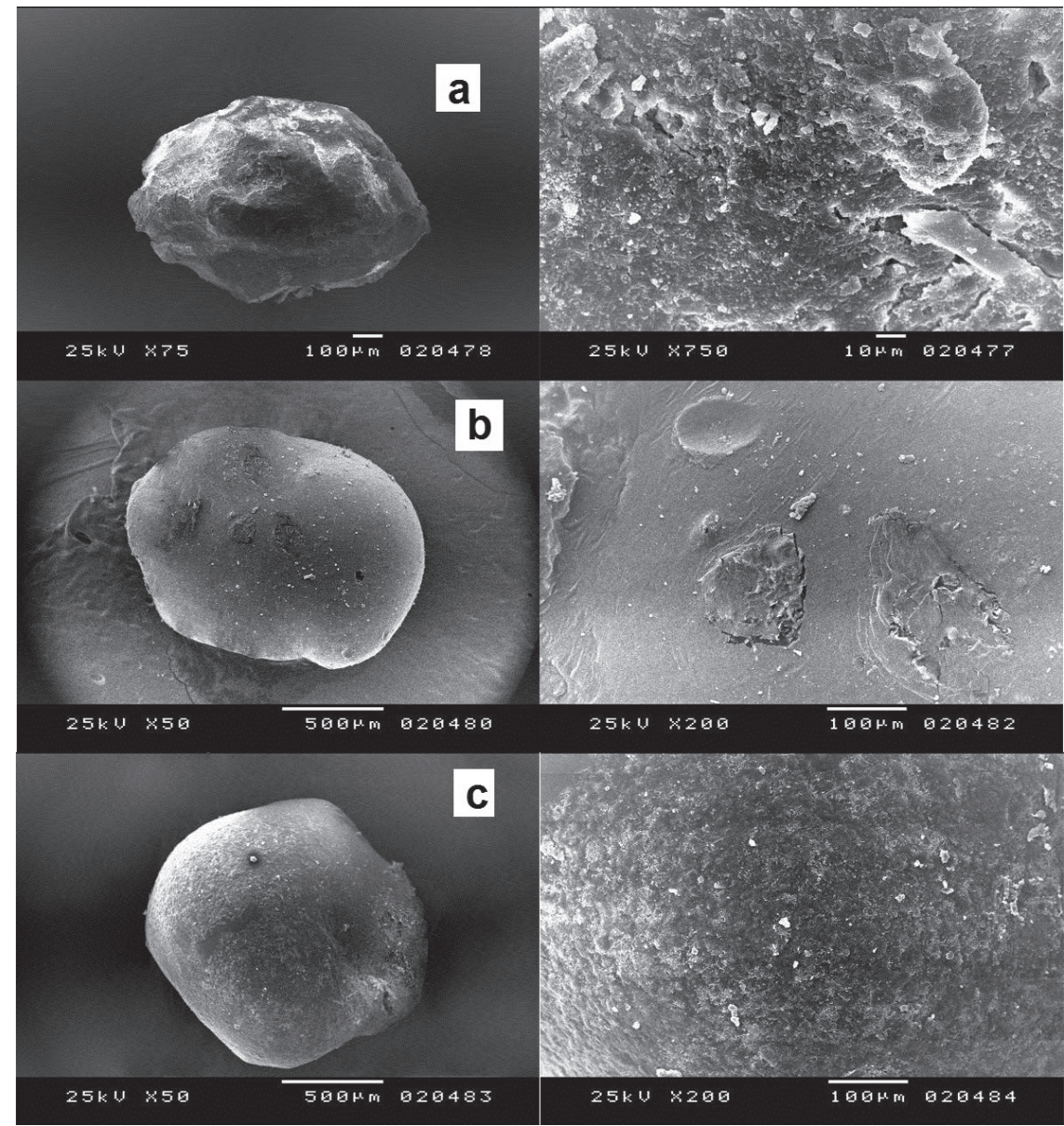

Fig. 1. Scanning electron micrographs of QT SLMPs a) Precirol, b) beeswax and c) Compritol.

their corresponding physical mixtures, indicating the presence of QT in the crystalline state dispersed in lipid matrices.

FTIR was performed to study the possibility of interaction between QT and the used lipids in the solid state (B1, C1 \& P1). IR spectrum of QT showed characteristic peaks at 3316 (O-H stretching), 3071 (C-H aromatic stretching), 2880 (C-H stretching), 1600 (C=O stretching) and $1340 \mathrm{~cm}^{-1}$ (C-H bending). IR spectra of the prepared solid lipid micro-pellets showed no appreciable change in the positions of characteristic bands of the drug, so it can be concluded that the drug maintains its identity without entering into any chemical interaction with the lipids used.

Drug content in the different batches of prepared SLMPs, equivalent to $10 \mathrm{mg}$ of the drug, was found to be in the range of $97.47 \pm 0.2 \%(n=30)$. Drug content values indicated high incorporation of QT in the lipophilic matrix with minimal loss. This can be attributed 
to the intense mixing and agitation during processing, causing suspended drug particles to de-aggregate in the molten polymer, resulting in a more uniform dispersion of fine particles in the lipophilic matrix.

In vitro release studies were performed in $0.1 \mathrm{~mol} \mathrm{~L}^{-1} \mathrm{HCl}(2 \mathrm{~h})$, followed by buffer solution of $\mathrm{pH}$ 6.8. The release data indicated that the release of QT was significantly dependent on the type of lipid used as a carrier. This difference in release profiles (Fig. 2) can be attributed to the chemical nature and the relative hydrophobicity of the lipids used (17). It was observed that as the hydrophobicity of the lipid increased, the QT release rate decreased. The highest QT release from the SLMPs was from the precirol containing formulation (90-99\%, Fig. 2c), then Precirol (50-77\%, Fig. 2b) and finally from beeswax formula-

a)

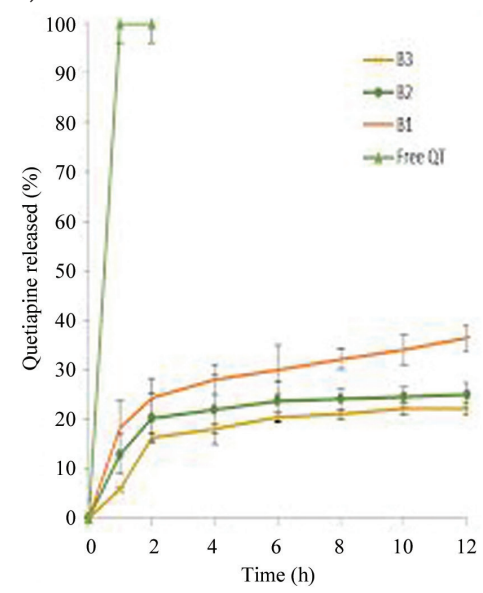

c)

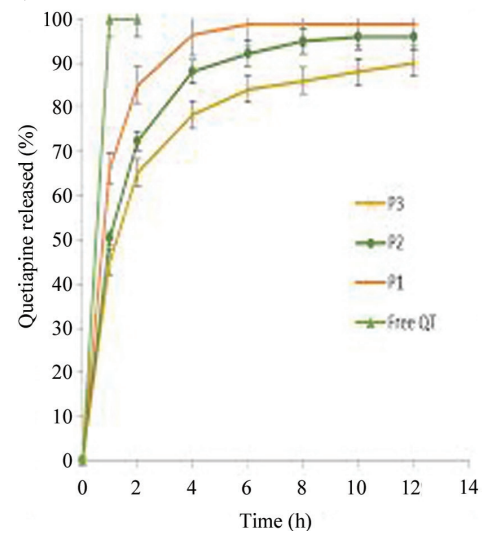

b)

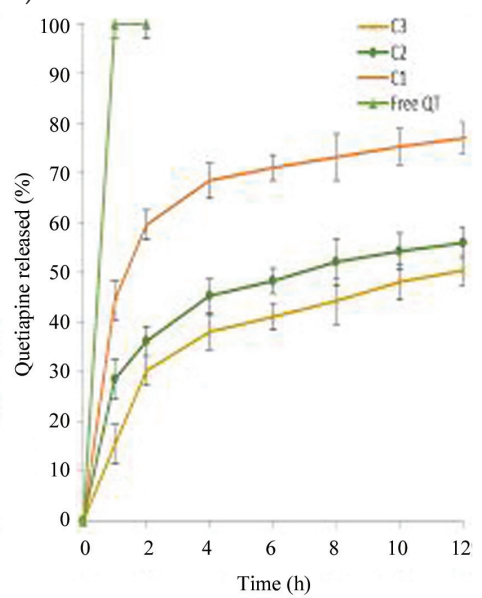

d)

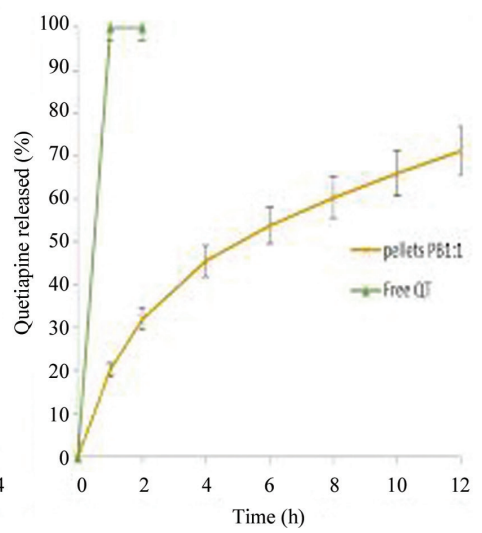

Fig. 2. Release profiles of QT from different SLMPs in $0.1 \mathrm{~mol} \mathrm{~L}^{-1} \mathrm{HCl}(2 \mathrm{~h})$, followed by $\mathrm{pH} 6.8$ buffer at $50 \mathrm{rpm}$ at $37 \pm 0.5^{\circ} \mathrm{C}$; a) beeswax, b) Compritol, c) Precirol and d) Precirol/beeswax 1:1, compared to that of free QT powder. 
A. Refaat et al.: A dual strategy to improve psychotic patients' compliance using sustained release quetiapine oral disintegrating tablets, Acta Pharm. 66 (2016) 515-531.

tion (22-36 \%, Fig. 2a). Moreover, surfactant properties of compritol and precirol led to faster release of QT compared to that from beeswax. In addition, precirol micropellets showed highly irregular surface with grooves and dentations in scanning electron (SE) micrographs (Fig. 1b), which may have contributed to the relatively faster QT release from precirol pellets (Fig. 2c).

SLMPs comprised of the three selected lipids shared a common property of significant abrupt release of QT in the first 2 hours of release in $0.1 \mathrm{~mol} \mathrm{~L}^{-1} \mathrm{HCl}$, followed by a relatively slower release rate in $\mathrm{pH}$ 6.8. This behavior may be attributed to the presence of drug crystals on the surface of the prepared micropellets and to the basic character of QT, whose solubilization is highly favored at acidic $\mathrm{pH}(6)$.

The release of QT from lipid micropellets was retarded by increasing the lipid to drug ratio (Fig. 2), which may be ascribed to the increased path length required for drug diffusion through the lipid matrix and increased time required for lipid matrix erosion, which will be illustrated later through modeling of release kinetics.

Since QT release from Precirol micropellets was very fast and that from beeswax micropellets very slow, micropellets with Precirol, beeswax and quetiapine in the ratio of 1:1:1 were prepared. The QT release profile is shown in Fig. 2d. The presence of Precirol accelerated drug release from the combination due to its surface active properties and its porosity imparting characteristic, which may enhance erosion of pellets and improve QT release.

Evaluation of release kinetics was performed by fitting the release data into kinetic models based on zero order, first order and Higuchi equations and then computing the correlation of determination $\left(R^{2}\right)$. A high $R^{2}$ value indicates applicability of a certain model to the release data.

Release data of QT from all the prepared SLMPs containing a single type of lipid showed biphasic release profiles: a relatively fast release phase followed by a slower one. These release profiles do not indicate zero order kinetics as confirmed by the relatively low values of the computed $R^{2}$.

On the other hand, release data from $\mathrm{PB}_{1: 1}$ was attributed to more or less equal release rate all over the data points, thus exhibiting sustained release behavior with zero order release $\left(R^{2}=0.999\right)$; it was therefore selected for further investigation and preparation of ODT.

In many release studies, the initial fast release phase was attributed to the release of surface drug before the matrix diffusion model took control (18) (as evident from the SEM). The slow release phase was attributed to the progressive drop in concentration gradient across the matrix through which the drug diffuses to the exterior (19).

Due to the chemical and physical complexity of lipophilic carriers, they may exhibit complex behavior (i.e., physical modifications on storage). Physical ageing effects associated with glyceride-based dosage forms have to be well understood in order to avoid any further drug release alteration during storage (20). Release stability profiles of the selected micro-pellet formula (PB) were performed over a one-year study period, under shelf storage conditions (room temperature $25^{\circ} \mathrm{C}$ and $25 \%$ relative humidity). Acceptable stability of this formula accompanied by a non-significant decrease $(p>0.05)$ in the overall release pattern of QT may be attributed to slight densification of polymer chains by storage.

Compression of multiparticulate units into ODT formulations remains challenging because of the high compression force required for tableting, which may cause damage to 
A. Refaat et al.: A dual strategy to improve psychotic patients' compliance using sustained release quetiapine oral disintegrating tablets, Acta Pharm. 66 (2016) 515-531.

the multiparticulate system and, as a result, compromise their release properties (21). Freeze drying is an alternative technique to produce ODTs without applying compaction force, which could be useful in the formulation of multiparticulate units as pellets into ODTs. However, some requirements need to be met in order to ensure development of a successful formulation. A liquid formulation is supposed to be viscous enough to suspend the multiparticulate unit long enough to complete formulation and freezing without compromising the disintegration performance. Minimum interaction between the liquid formulation and the multiparticulate unit should be attained to avoid unwanted changes in the original properties of the multiparticulate one such as early drug leakage (22).

Croscarmellose is a FDA-approved disintegrant that has been reported as a matrix former in the production of lyophilized ODT, where its amorphous polymeric structure imparts strength and resilience while retaining some flexibility and ensuring a smooth, rapid melt in the mouth (23). Moreover, its superdisintegrant properties help achieve rapid disintegration of the prepared tablets. PVP-K25 is used in our formulations as a viscosity imparting agent to help suspending pellets during formulation and freezing (24).

Mannitol is a commonly used sugar alcohol in the preparation of lyophilized ODT (23). Its crystallization during freezing provides rigidity. The presence of mannitol in the formulation of freeze-dried ODTs has been reported to function not only as a matrix supporting agent/structure former, but also as a disintegration enhancing agent (25). Therefore, mannitol has dual functions as an excipient, not only to influence the mechanical properties of the tablets by imparting hardness but also, owing to its highly hydrophilic nature, to enhance rapid disintegration of the tablets.

Viscosity measurement of matrices' liquid blends revealed that increasing the concentration of PVP increased the viscosity of formulations containing the same amount of mannitol, which is attributed to the viscosity imparting properties of PVP (Table IV). It was also observed that formulations containing higher amounts of mannitol showed higher viscosity values, which could be due to the increased amount of solute in the formulations, and therefore increased intermolecular interactions strengthening the binder matrix of the tablets, synergizing the effect of PVP as a binder.

Formulations $\mathrm{M}_{7}, \mathrm{M}_{8}$ and $\mathrm{M}_{9}$ showed the highest hardness values (Table IV), which may be ascribed to the high percentage of mannitol, which affected hardness by increasing the amount of solute in the formulations. Therefore, an increase in intermolecular interactions occurred and strengthened the binder matrix of the tablets, synergizing the effect of PVP as a binder. Similar results were reported when gelatin was used with carrageenan (26).

Studies investigating optimization of the concentration of matrix supporting agents in the formulation of freeze-dried ODTs have been previously reported. Chandrasekhar et al. investigated the influence of mannitol, sorbitol and sucrose concentrations on the hardness and disintegration times of the tablets (27). AlHusban et al. investigated mannitol concentrations of 10, 30, 50 and $70 \%$ (of total solid material) (28). They observed that mannitol maintained an amorphous state at 10 and $30 \%$ (of total solid material), whilst it underwent crystallization at 50 and $70 \%$ of total solid material, which could explain the increase in tablet hardness compared to those with 10-30 \% mannitol concentration.

In the present study, high mannitol concentrations (45-60\%) were utilized to promote its crystallization and hence produce robust ODT matrices. Also, PVP percentage influenced the hardness of formulations containing the same amount of mannitol due to the binding properties of PVP. 
In general, ODTs manufactured using freeze-drying exhibit rapid disintegration and dissolution due to their highly porous nature, which allows penetration of saliva into the matrix of the tablets, resulting in disintegration (29). There is no USP in vitro method to evaluate the disintegration time for ODTs that would represent the in vivo disintegration time in the mouth. Therefore, FDA recommends using a modified form of the USP disintegration test (30), which may be the wetting test. Results obtained from the wetting test are shown in Table IV, along with oral disintegration data. A good correlation between oral disintegration time and wetting time was obtained for different lyophilized ODT matrices. This indicates that the wetting time test can be considered as a modified USP disintegration test that could be used in quality control testing of ODT, since oral disintegration time measurement is not practical for routine quality control work.

Comparing the viscosities of matrix solutions with hardness and disintegration data (Table IV), it was observed that formulations with lower viscosity values and lower mannitol content $\left(\mathrm{M}_{1}, \mathrm{M}_{2}\right.$ and $\left.\mathrm{M}_{3}\right)$ exhibited rapid oral disintegration times but the lyophilized matrices' tablets were soft and fragile. On the other hand, formulations with higher viscosity values and high mannitol content $\left(\mathrm{M}_{7}, \mathrm{M}_{8}\right.$ and $\left.\mathrm{M}_{9}\right)$ showed the most delayed oral disintegration times, which is attributed to the formation of a viscous solution upon hydration on the tongue, reducing the rate of saliva penetration through the tablet and resulting in longer disintegration time (22). Further, formulations with the highest viscosity values showed high hardness values (Table IV). The increased amount of solute in these formulations may increase intermolecular interactions, resulting in the strengthening of the binder matrix of the tablets.

Scanning electron micrographs were utilized to study surface properties and porosity of the prepared tablet matrices. The study was performed on three different matrix formulations, namely $\mathrm{M}_{1}$ (exhibiting the most rapid disintegration time), $\mathrm{M}_{9}$ (with the most delayed disintegration time) and $\mathrm{M}_{4}$ (with intermediate disintegration time) (Table IV). A common observation was the presence of mannitol crystals on the surface (Fig. 3a) and in the core of the analyzed tablet matrices, which is attributed to the high percentage mass of

Table IV. Physical characteristics of lyophilized ODT matrices

\begin{tabular}{ccccc}
\hline $\begin{array}{c}\text { Matrix } \\
\text { code }\end{array}$ & $\begin{array}{c}\text { Mean viscosity } \\
(\mathrm{mPa})\end{array}$ & $\begin{array}{c}\text { Mean hardness } \\
(\mathrm{N})\end{array}$ & $\begin{array}{c}\text { Wetting time } \\
(\mathrm{s})\end{array}$ & $\begin{array}{c}\text { Oral disintegration } \\
\text { time }(\mathrm{s})\end{array}$ \\
\hline $\mathrm{M}_{1}$ & $125.3 \pm 2.1$ & $8.8 \pm 2.0$ & $20.1 \pm 2.3$ & $10.2 \pm 1.2$ \\
$\mathrm{M}_{2}$ & $132.4 \pm 1.9$ & $10.0 \pm 2.4$ & $24.2 \pm 2.7$ & $13.4 \pm 3.2$ \\
$\mathrm{M}_{3}$ & $145.2 \pm 1.6$ & $14.2 \pm 1.5$ & $35.2 \pm 2.4$ & $20.2 \pm 3.1$ \\
$\mathrm{M}_{4}$ & $134.5 \pm 2.3$ & $17.8 \pm 1.3$ & $40.3 \pm 2.9$ & $21.5 \pm 2.1$ \\
$\mathrm{M}_{5}$ & $141.6 \pm 2.7$ & $20.6 \pm 1.1$ & $48.1 \pm 3.2$ & $25.1 \pm 4.1$ \\
$\mathrm{M}_{6}$ & $156.2 \pm 1.2$ & $25.2 \pm 3.2$ & $54.3 \pm 3.3$ & $32.3 \pm 4.9$ \\
$\mathrm{M}_{7}$ & $157.1 \pm 1.4$ & $27.1 \pm 3.7$ & $70.1 \pm 3.1$ & $50.1 \pm 3.3$ \\
$\mathrm{M}_{8}$ & $165.6 \pm 1.2$ & $30.6 \pm 4.3$ & $84.2 \pm 1.7$ & $58.4 \pm 1.5$ \\
$\mathrm{M}_{9}$ & $185.1 \pm 0.9$ & $35.2 \pm 4.1$ & $95.2 \pm 1.4$ & $70.1 \pm 2.4$ \\
\hline
\end{tabular}


A. Refaat et al.: A dual strategy to improve psychotic patients' compliance using sustained release quetiapine oral disintegrating tablets, Acta Pharm. 66 (2016) 515-531.

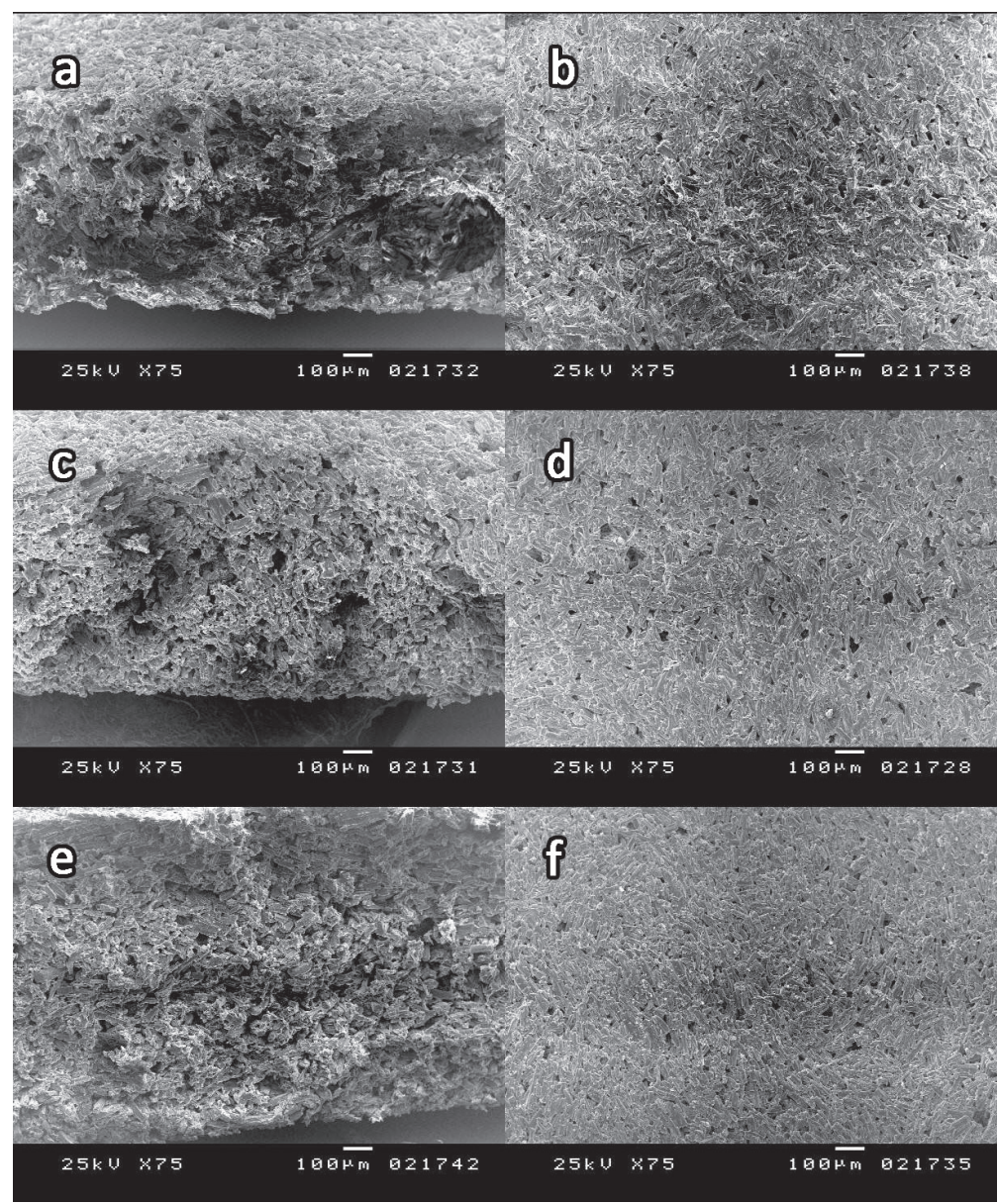

Fig. 3. SE micrographs for the external surface of a) M1, c) M4 and e) M9 along with surface structure of b) M1, d) M4, and f) M9.

mannitol used in all formulations, higher than $30 \%$ of the matrix dispersion mass. Similar results were reported by Al Husban et al. (27).

Matrix formulation $\mathrm{M}_{1}$ (Fig. $3 \mathrm{~b}$ ) showed a highly porous structure that may have been responsible for its rapid oral disintegration time $(10 \mathrm{~s})$, while the $\mathrm{M}_{9}$ matrix formulation showed a decrease in the size and density of pores (Fig. 3d), which was reflected in its oral disintegration time (70 s) (Table IV). The possible explanation of this observation is that the inclusion of a higher solid content in formulation $\mathrm{M}_{9}$ cemented the porous framework provided by the water soluble polymer, croscarmellose and PVP, and therefore the porous space in the tablets was reduced, which led to a reduction in tablet porosity (22).

It is worth concluding that the ODT disintegration behavior may be influenced by many factors, including viscosities of the matrix solution, hardness as well as thickness 
and porosity of the prepared ODT solid matrix. $\mathrm{M}_{9}$ showed the most delayed oral disintegration time, which could be related to many observations as the formation of a viscous solution upon hydration on the tongue and the high hardness values of the matrix formed (Table IV). In addition, the reduced porosity of this formula, $\mathrm{M}_{9}$, as shown by SEM micrographs (Fig. 3d), may also induce delayed oral disintegration.

For preparation of different QT tablet matrices, the optimal matrix composition that yields tablets with minimal oral disintegration time and maximal hardness was chosen. $\mathrm{M}_{4}$ formulation was selected for further studies. The viscosity of its liquid blend allowed easy dosing into the PVC blisters forming intact tablets after lyophilization. $\mathrm{M}_{4}$ lyophilized tablets are characterized by a good balance between hardness $(17.8 \mathrm{~N})$ and oral disintegration time (21.5 s) (Table IV), relatively hard and robust structure and easy extraction from PVC packs. $\mathrm{M}_{1}, \mathrm{M} 2$ and $\mathrm{M}_{3}$ tablet formulations were soft and somewhat fragile and not easily extracted from blisters. $\mathrm{M}_{7}, \mathrm{M}_{8}$ and $\mathrm{M}_{9}$ liquid blends were not easily dosed into PVC blisters due to their high viscosities (Table IV). Lyophilized tablets were hard and stuck to the PVC packs showing the most delayed oral disintegration times (50, 58 and 70 s, respectively); thus they were excluded from our further investigations.

Preliminary studies were performed to find the most suitable ratio of matrix formulation $\left(\mathrm{M}_{4}\right)$ to micropellets, required for suspending the micropellets during preparation, and ratio 2:1 was selected for further studies.

As discussed before, micropellets (PB) showed sustained release behavior with zero order release, which did not change significantly $(p>0.05)$ upon storage, and that is why they were selected to be incorporated in the lyophilized matrices to prepare QT ODT formulations.

Solution of the $\mathrm{M}_{4}$ formulation was easily blended with PB micropellets, where the amount of matrix solution and its viscosity were sufficient to suspend pellets during preparation and freeze drying. Intact, relatively hard tablets were obtained and were easily extracted from blisters, showing homogenous distribution of the micropellets through the matrix.

Physical characteristics of the prepared QT ODTs were investigated for both $\mathrm{F}_{1}$ and $\mathrm{F}_{2}$ formulations. They showed average masses of $314 \pm 2$ and $627 \pm 4 \mathrm{mg}$, average hardness of

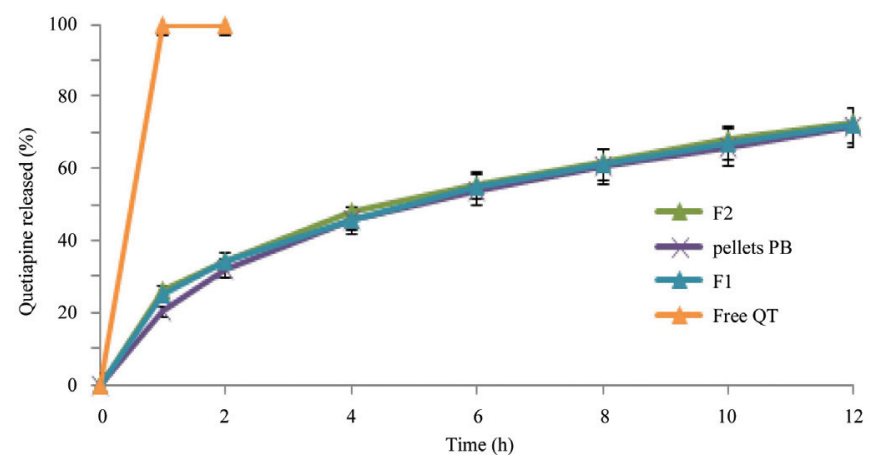

Fig. 4. Release profiles of QT from F1 and F2 and PB micropellets compared to free QT powder in 0.1 $\mathrm{mol} \mathrm{L}-1 \mathrm{HCl}(2 \mathrm{~h})$, followed by $\mathrm{pH} 6.8$ buffer at $50 \mathrm{rpm}$ at $37 \pm 0.5^{\circ} \mathrm{C}$. 
$16.5 \pm 2.3$ and $17.3 \pm 2.2 \mathrm{~N}$, average oral disintegration times of $19.1 \pm 3.4$ and $23.9 \pm 4.1 \mathrm{~s}$ and average wetting times of $36.9 \pm 3.6$ and $45.0 \pm 5.1 \mathrm{~s}$, respectively. It was observed that hardness, oral disintegration and wetting data were not significantly different $(p>0.05)$ from those of the $\mathrm{M}_{4}$ matrix. Acceptable taste was reported by all volunteers in oral disintegration testing.

Fig. 4 shows the rate of QT release from $\mathrm{F}_{1}$ and $\mathrm{F}_{2}$ compared to that from PB micropellets, where release profiles were not significantly different (Student $t$-test, $p>0.05$ ).

Release of QT from the prepared ODT was slightly faster during the first hour, which may be attributed to the release of surface QT from micropellets into the tablet matrix during the lyophilization step.

\section{CONCLUSIONS}

Lipid carriers can be utilized to prepare QT micropellets with a high drug content and acceptable yield using the hot extrusion method, which can successfully achieve sustained release and taste masking effect of the drug at a relatively low cost. Release pattern of QT from the prepared solid lipid micropellets was dependent on the type of lipid carrier; they exhibited the most rapid release with Precirol and the most sustained with beeswax. A tertiary system of Precirol/beeswax/QT in 1:1:1 ratio showed an intermediate release pattern with high $R^{2}$ values of a zero order model with reduced burst release. This release pattern was not significantly changed after 12 months of shelf storage.

Freeze drying is a promising alternative technique to direct compression in the formulation of multiparticulate units into ODTs without application of any compaction force. The selected lyophilized matrix formulation $\left(\mathrm{M}_{4}\right)$ containing $50 \%$ mannitol, $2 \%$ croscarmellose and $2.5 \%$ PVP showed superior wetting time of $41 \mathrm{~s}$, along with excellent oral disintegration time of $22 \mathrm{~s}$, with acceptable hardness $(16.85 \mathrm{~N})$ and suitable viscosity to suspend pellets during the freezing process. ODTs containing QT lipid pellets are promising delivery systems that provide enhanced compliance for psychotic patients.

\section{REFERENCES}

1. www.accessdata.fda.gov/drugsatfda.../97/020639ap_Seroquel_apltr.pdf. (Access date November 22, 2015).

2. M. A. Kondo, K. Tajinda and C. Colantuoni, Unique pharmacological actions of atypical neuroleptic quetiapine: possible role in cell cycle/fate control, Transl. Psychiatry 3 (2013) 2-43; DOI: 10.1038/tp.2013.19.

3. J. Locklear, H. Svedsäter. C. Datto and J. Endicott, Effects of once-daily extended release quetiapine fumarate (quetiapine XR) on quality of life and sleep in elderly patients with major depressive disorder, J. Affect. Disord. 149 (2013) 189-195; DOI: 10.1016/j.jad.2013.01.021.

4. C. Cecchic and P. Canonico, Pharmaceutical formulations and adherence to pharmacological treatment in psychiatry: the example of oral disintegrating tablet of olanzapine, Riv. Psichiatr. 47 (2012) 30-39; DOI: 10.1708/1034.11289.

5. S. K. Srivastava, R. Verma, V. Chandra and S. P. Srivastava. Orally disintegrating tablets: A dosage form that extends the market exclusivity and patient protection, World J. Pharmacy Pharm. Sci. 3 (2014) 526-546. 
A. Refaat et al:: A dual strategy to improve psychotic patients' compliance using sustained release quetiapine oral disintegrating tablets, Acta Pharm. 66 (2016) 515-531.

6. http://reference.medscape.com/drug/seroquel-xr-quetiapine-342984\#10 (Access date November 22, 2015).

7. L. V. Allen, N. G. Popovich and H. C. Ansel, Solid Dosage Forms and Solid Modified Release Delivery Systems, in Ansel's Pharmaceutical Dosage Forms and Drug Delivery Systems, Lippincott Williams \& Wilkins, Philadelphia 2005.

8. G. M. Clarke and J. M. Newton, Short, comparative gastrointestinal transit of pellet systems of varying density, Int. J. Pharm. 114 (1995) 1-11; DOI: 10.1016/0378-5173(94)00200-O.

9. J. Collett and C. Moreton, Modified-Release per Oral Dosage Forms, in Pharmaceuticals: the Science of Dosage Form Design (Ed. M. E. Aulton), Churchill Livingstone, New York 2001, pp. 289-305.

10. M. K. Raval, R. V. Ramani and N. R. Sheth, Formulation and evaluation of sustained release entericcoated pellets of budesonide for intestinal delivery, Int. J. Pharm. Investig. 3 (2013) 203-211. DOI: 10.4103/2230-973X.121294.

11. M. H. Kumar, K. Samatha, A. Balaji and M. S. Shankar, Recent novel advancements in pellet formulation. A Review, Int. J. Pharm. Sci. Res. 4 (2013) 3803-3822; DOI: 10.13040/IJPSR.0975-8232.4 (10).3803-22.

12. Hoda Maher El Taher, Development of Pelletization Technology for the National Pharmaceutical Industry, Master thesis, Faculty of Pharmacy, Alexandria University.

13. S. El Gamal, V. Naggar and M. Sokar, Colon targeting of mebeverine $\mathrm{HCl}$ from $\mathrm{pH}$-dependent tablet formulations, J. Am. Sci. 8 (2012) 632-638.

14. P. Dey and S. Maiti, Orodispersible tablets: A new trend in drug delivery, J. Nat. Sci. Biol. Med. 1 (2010) 2-5; DOI: 10.4103/0976-9668.71663.

15. S. Sherimeier and P. C. Schmidt, Fast dispersible ibuprofen tablets, Eur. J. Pharm. Sci. 15 (2002) 295-305; DOI: 10.1016/S0928-0987(02)00011-8.

16. C. Reitz and P. Kleinebudde, Solid lipid extrusion of sustained release dosage forms, Eur. J. Pharm. Biopharm. 67 (2007) 440-448; DOI: 10.1016/j.ejpb.2007.03.008.

17. C. M. Adeyeye and J. C. Price, Development and evaluation of sustained-release ibuprofen-wax microspheres. In vitro dissolution studies, Pharm. Res.11(1994)575-579; DOI:10.1023/A:1018931002991.

18. J. B. Schwartz, A. P. Simonelli and W. I. Higuchi, Drug release from wax matrices. I. Analysis of data with first-order kinetics and with the diffusion-controlled model, J. Pharm. Sci. 57 (1968) 274277; DOI: 10.1002/jps.2600570206.

19. R. Senjković and I. Jalšenjak, Apparent diffusion coefficient of sodium phenobarbitone in ethylcellulose microcapsules: Effects of capsule size, J. Pharm. Pharmacol. 33 (1981) 279-282; DOI: 10.1111/ j.2042-7158.1981.tb13781.x.

20. J. Hamdani, A. J. Moes and K. Amighi, Physical and thermal characterisation of Precirol ${ }^{\circledR}$ and Compritol $^{\circledR}$ as lipophilic glycerides used for the preparation of controlled-release matrix pellets, Int. J. Pharm. 206 (2003) 47-57; DOI: 10.1016/S0378-5173(03)00229-1.

21. R. Bodmeier, Tableting of coated pellets, Eur. J. Pharm. Biopharm. 43 (1997) 1-8; DOI: 10.1016/S09396411(96)00028-8.

22. F. Al Husban, Y. Perrie and A. R. Mohamed, Formulation of multiparticulate systems as lyophilised orally disintegrating tablets, Eur. J. Pharm. Biopharm. 79 (2011) 627-634; DOI: 10.1016/j.ejpb.2011.05.014.

23. F. Lai, E. Pini, F. Corrias, J. Perricci, M. Manconi, A. M. Fadda and C. Sinico, Formulation strategy and evaluation of nanocrystal piroxicam orally disintegrating tablets manufacturing by freezedrying, Int. J. Pharm. 467 (2014) 27-33; DOI: 10.1016/j.ijpharm.2014.03.047.

24. http://www.brenntagspecialties.com/en/downloads/Products/Multi_Market_Principals/Ashland/ PVP_-_PVP_VA/PVP_Brochure.pdf (access date November 22, 2015).

25. A. K. Nayak and K. Manna, Current developments in orally disintegrating tablet technology, J. Pharm. Educ. Res. 2 (2011) 1-10. 
26. C. Michon, B. Cuvelier, B. Launay, A. Parker and G. Takerkart. Study of the compatibility/incompatibility of gelatin/iota-carrageenan/gelatin/water mixtures, Carbohydr. Polym. 28 (1995) 333-336; DOI: 10.1016/0144-8617(95)00096-8.

27. R. Chandrasekhar, Z. Hassan, A. M. Smith and F. Al Husban, The role of formulation excipients in the development of lyophilized fast-disintegrating tablets, Eur. J. Pharm. Biopharm. 72 (2009) 119129; DOI: 10.1016/j.ejpb.2008.11.011.

28. F. Al Husban, Y. Perrie and A. R. Mohammed, Preparation, optimisation and characterization of lyophilized rapid disintegrating tablets based on gelatin and saccharide, Curr. Drug Deliv. 7 (2010) 65-75; DOI: 10.2174/156720110790396427.

29. X. Tang and M. J. Pikal, Design of freeze-drying processes for pharmaceuticals: practical advice, Pharmaceut. Res. 21 (2004) 191-200; DOI: 10.1023/B:PHAM.0000016234.73023.75.

30. Guidance for Industry: Orally Disintegrating Tablets, FDA CDER Data Standards Manual, 2008. 
